\title{
A Two Population Model of Cancer Growth With Fixed Carrying Capacity
}

\author{
Stura Ilaria*, Gabriele Domenico*, Guiot Caterina*
}

\begin{abstract}
Cancer is not a homogenous tissue, but a very complex mix of different cell populations; moreover, a delicate equilibrium exists between these components of tumor mass. In this work we address prostate cancer although the methods presented can be generalized to most tumour types. The aim of our work is to model the behaviour of the different cell populations within the tumour and simulate changes which occur during natural evolution and treatments.
\end{abstract}

\section{INTRODUCTION}

Prostate cancer is the most prevalent tumor affecting the male population and in approximately $30 \%$ of cases it develops chronically for a long period of time (i.e. years), requiring surgical and/or medical interventions (e.g. Androgen-Deprivation Therapies with anti-androgens drugs or LHRH analogues). Usually, the tumor reacts to the hormonal therapy for a highly variable time span, from some months to more than ten years, finally becoming hormone-resistant. This behavior is related to the co-existence in prostate cancer of cells with a different sensibility to hormones: in particular, simplifying the cellular spectrum, we may suppose a balance between two groups of hormone-sensitive versus hormone-resistant cells. Under the pressure of an anti-androgen drug, the second group becomes gradually dominant and causes the clinical relapse and the emergence of a disseminated castration-resistant prostate cancer.

Two main models were developed to depict the evolution of the ratio between the sensitive and resistant cells: the 'adaptation' model and the 'clonal selection' model ([1],[2],[3]). The adaptation model suggests that prostate cancer is initially composed by homogeneous cells, in terms of their androgen requirement, and castration resistance emerges through genetic or epigenetic switches of androgendependent cells to androgen-independent cells. On the contrary, the 'clonal selection' model states that primary prostate cancer cells are heterogeneous regarding their androgen requirement, and so a minority of them is a clone of pre-existing castrationresistant cells. In an androgen-deprived environment, these castration-resistant cells are selected for their survival and proliferative advantage.

Our model describes such scenario and simulates interactions between clones taking into account that the general amount of nutrients and the anatomical space available are limited (fixed carrying capacity) and the different effects that treatments have on various cell populations. In section II there is a brief introduction on the Phenomenological Universalities approach (or PUN) to simulate a cancer cell population, in III we will explain how to model the tumor as two populations system and in III-B our results on how the behaviour of a population could change in relation to the other one are reported.

\section{PUN}

The Phenomenological Universalities (PUN) approach (see [4], [5] for details) describes tumor growth with the equations:

$$
\left\{\begin{array}{l}
\frac{d N(t)}{d t}=c(t) N(t) \\
\frac{d c(t)}{d t}=\sum_{i=0}^{n} \beta_{i} c^{i}
\end{array}\right.
$$

$N$ is the cancer cells population numerosity, $c(t)$ is the growth rate function and $n$ is the degree of its Taylor expansion. This approach generalizes the most used equations in population growth, in fact: in the case $n=0, c(t)$ constant, $N$ grows as an exponential law; for $n=1$, as a gompertzian law and for $n=2$ as West/logistic growth law.

We focus our attention on the case $n=1$ (gompertzian growth law): this function has an exponential initial growth that progressively tends to slow and finally it reaches its carrying capacity. This behavior reflects very realistically the trend of a tumor: in an early stage, cells grow faster because they have a lot of nutrient and space, than the cancer core becomes necrotic and hypoxic and/or the tumor reaches some physical barrier such as tissue or bones and it can't swell again. The dynamic system, in $U 1$ case, is:

$$
\left\{\begin{array}{l}
\frac{d N(t)}{d t}=c(t) N(t) \\
\frac{d c(t)}{d t}=\beta c
\end{array}\right.
$$


or

$$
\frac{d N(t)}{d t}=c_{0} e^{\beta t} N(t)
$$

The solution of (2) is:

$$
N(t)=N_{0} e^{\frac{c_{0}}{\beta}\left(e^{\beta t}-1\right)}
$$

Being $\beta$ inversely proportional to the carrying capacity that tumor can reach and $c_{0}$ the growth rate. Note that in this case the carrying capacity depends on the initial condition $N_{0}$ : we will normalize this equation as:

$$
V(t)=\frac{N(t)}{N_{0}}=e^{\frac{c_{0}}{\beta}\left(e^{\beta t}-1\right)}
$$

To stress the importance of the carrying capacity, equation (3) can be rewritten as:

$$
N(t)=N_{\infty} e^{z e^{-r t}}
$$

where $N_{\infty}$ is the carrying capacity and $r$ the growth rate. We can easily transform (3) in (4) and vice versa with $r=-\beta, N_{\infty}=N_{0} e^{-\frac{c_{0}}{\beta}}$ and $z=\frac{c_{0}}{\beta}$. In the next section we will show a two populations model based on PUN.

\section{TWO POPULATIONS MODEL}

Two different populations of cells, due to genetic (e.g. two clones of the same tumor) or epigenetic (e.g. necrotic center of the mass) relation, are considered and described using PUN approach.

If the two populations are gompertzian and independent to each other, the system becomes:

$$
\left\{\begin{array}{l}
\frac{d N_{1}(t)}{d t}=c_{1}(t) N_{1}(t) \\
\frac{d N_{2}(t)}{d t}=c_{2}(t) N_{2}(t)
\end{array}\right.
$$

and solutions are independent, so each population grows as in (1). The only constraint will be that the total amount of the carrying capacities is a constant: $N_{\infty, 1}+N_{\infty, 2}=N_{\infty}$, being $N_{\infty, 1}$ and $N_{\infty, 2}$ the carrying capacities of $N_{1}$ and $N_{2}$ respectively in the system (5).

\section{A. The two populations model with mutation}

A more complex system, where population 1 could mutate in 2 but 2 couldn't mutate in 1 , as occurs, for example, if 2 is a more resistant and/or more aggressive clone than 1 , is described by:

$$
\left\{\begin{array}{l}
\frac{d N_{1}(t)}{d t}=c_{1}(t) N_{1}(t)-m N_{1}(t) \\
\frac{d N_{2}(t)}{d t}=c_{2}(t) N_{2}(t)+m N_{1}(t)
\end{array}\right.
$$

where $m$ is the mutation rate.
1) Analytical solutions: The first equation has no dependencies from the second, so we can find easily the solution $N_{1}=e^{\int^{t} c_{1}(s)-m d s}$. The second equation depends both on $N_{1}$ and $N_{2}$ and is a linear non homogeneous equation:

$N_{2}=e^{\int^{\lambda} c_{2}(s) d \lambda}\left[\int^{t} e^{\int^{\lambda} c_{2}(\epsilon) d \epsilon} m e^{\int^{\lambda} c_{1}(\epsilon)-m d \epsilon} d \lambda+C\right]$

Note that in some cases $N_{2}$ does not have a closed form, but it could be approximated with numerical analysis tool, e.g. MATLAB (MATLAB and Statistics Toolbox 7.0, The MathWorks, Inc., Natick, Massachusetts, United States.) or Octave.

2) Equilibrium points: Forcing equations (6) to zero and solving the system, we could easily find that the equilibrium points are $P_{1}=(0,0)$ and $P_{2}=\left(n_{1}, \frac{-m n_{1}}{c_{2}(t)}\right)$, with $N_{1}\left(t^{*}\right)=n_{1}$ and $c\left(t^{*}\right)=m$; by studying the Jacobian matrix and the eigenvalues $\lambda_{1,2}$, we know that points are stable or unstable depending on $\Delta=\left(c_{2}(t)-c_{1}(t)+m\right)^{2}$ and $\lambda_{1,2}$ :

1) if $\Delta=0$ then $\lambda_{1,2}=c_{2}(t) \Rightarrow \lambda_{1,2}>0 \Rightarrow$ unstable point

2) if $\Delta>0$ then $\lambda_{1} \neq \lambda_{2}$ and $\lambda_{1,2} \in \mathbf{R}$

3) if $\lambda_{1,2}>0 \Rightarrow$ unstable point

4) if $\lambda_{1,2}<0 \Rightarrow$ stable point

5) if $\lambda_{1}<0, \lambda_{2}>0$ or $\lambda_{1}>0, \lambda_{2}<0 \Rightarrow$ saddle point (unstable)

3) U1 case: In case of U1 (gompertzian growth law) we have $c_{1}(t)=c_{1} e^{\beta_{1} t}, c_{2}(t)=c_{2} e^{\beta_{2} t}$ with $c_{1}, c_{2}, \beta_{1}$ and $\beta_{2}$ constants.

The analytical solutions are

$$
\begin{aligned}
N_{1} & =e^{\int^{t} c_{1}(s)-m d s}=N_{0} e^{\frac{c_{1}}{\beta_{1}} e^{\beta_{1} t}-m t-\frac{c_{1}}{\beta_{1}}}= \\
& =N_{\infty, 1} e^{-r_{1} e^{\frac{t}{N_{\infty}, 1}}-m t+z_{1}} \\
N_{2} & =e^{\int^{\lambda} c_{2}(s) d \lambda}\left[\int^{t} e^{\int^{\lambda} c_{2}(\epsilon) d \epsilon} m e^{\int^{\lambda} c_{1}(\epsilon)-m d \epsilon} d \lambda+C\right]= \\
& =e^{c_{2} e^{\beta_{2} t}-c_{2}}\left(m \int^{t} e^{-\frac{c_{2}}{\beta_{2}} e^{\beta_{2} \lambda}+\frac{c_{2}}{\beta_{2}}} N_{1}(\lambda) d \lambda+C\right)
\end{aligned}
$$

The equilibrium points are $P_{1}=(0,0), P_{2}=$ $\left(N_{1}\left(t^{*}\right), \frac{-m N_{1}\left(t^{*}\right)}{c_{2} e^{\beta_{2} t^{*}}}\right)$ and they all are unstable.

Studying the limiting behaviour for $t \rightarrow \infty$, we can see that $N_{1}$ tends to zero when $m>0$; for $N_{2}$ it is too complicate to find a close solution and its relative limit, but plotting the solution using MATLAB we saw that the limit of $N_{2}$ depends to $\frac{c_{1} c_{2}}{\beta_{1} \beta_{2}}$.

Re-writing the system using eq. (4) we have:

$$
\left\{\begin{array}{l}
\frac{d N_{1}(t)}{d t}=-r_{1} N_{1}(t) \log \left(\frac{N_{1}}{N_{\infty, 1}}\right)-m N_{1}(t) \\
\frac{d N_{2}(t)}{d t}=-r_{2} N_{2}(t) \log \left(\frac{N_{2}}{N_{\infty, 2}}\right)+m N_{1}(t)
\end{array}\right.
$$


In this case $N_{1}=N_{\infty, 1} e^{-\frac{m}{r_{1}}} e^{\log \left(\frac{y_{0}}{N_{\infty, 1}}\right) e^{\frac{m}{r_{1}}-\frac{r_{1} t}{1-m}}}$ which tends, for $t \rightarrow \infty$, to the new carrying capacity $N_{\infty, 1}^{*}$ that depends on the old ones $N_{\infty, 1}$ and on the mutation rate: $N_{\infty, 1}^{*}=N_{\infty, 1} e^{-\frac{m}{r_{1}}}$.

The carrying capacity of the mutated population $N_{2}$ is $N_{\infty, 2}^{*}=\frac{m k e^{-\frac{m_{1}}{r_{1}}}}{r_{2} \log \left(\frac{N_{2}^{*}}{N_{\infty}}\right)}$, where $N_{2}^{*}=N_{2}\left(t^{*}\right)$ and $t^{*}$ is the time in which $N_{1}$ reaches its own carrying capacity $N_{\infty, 1}^{*}$. We can see in the phase portrait that the system tends to the equilibrium point $\left(N_{1}^{*}, N_{2}^{*}\right)$; other variations of the parameters do not lead to a phase change.

\section{B. Two populations model following treatments}

When the cancer is detected in a patient, in the majority of cases the tumor will be treated to reduce its volume. There is a large variety of treatments: surgery, chemotherapy, radiotherapy and hormone therapy are the most common. We suppose that the two populations will respond in different manners to a treatment, in particular we assume the cell population 1 to be very sensitive and population 2 to be less sensitive or not sensitive at all (e.g. clone resistant to hormone therapy or a metastasis that will not be eradicated during surgery). The system (5) becomes:

$$
\left\{\begin{array}{l}
\frac{d N_{1}}{d t}=c_{1}(t) N_{1}-d_{1} N_{1} \\
\frac{d N_{2}}{d t}=c_{2}(t) N_{2}-d_{2} N_{2}
\end{array}\right.
$$

where $d_{1}$ and $d_{2}$ are the kill rates of the treatment on the populations 1 and 2 respectively; $d_{1}>>$ $d_{2}$ because 1 is more sensitive and $d_{2}$ could even vanish if the treatment has no effect of 2 .

1) Analytical solutions: The equations are independent to each other and have the same solutions of the first equation of (6):

$$
N_{1}=e^{\int^{t} c_{1}(s)-d_{1} d s} \quad N_{2}=e^{\int^{t} c_{2}(s)-d_{2} d s}
$$

2) Equilibrium points: The equilibrium points are $P_{1}=(0,0), P_{2}=\left(0, N_{2}^{*}\right), P_{3}=\left(N_{1}^{*}, 0\right)$ and $P_{4}=\left(N_{1}^{*}, N_{2}^{*}\right)$ with $N_{1}^{*}=N_{1}\left(t_{1}^{*}\right)$ where $t_{1}^{*}$ is the time in which $c_{1}\left(t_{1}^{*}\right)=d_{1}$ and $N_{2}^{*}=N_{2}\left(t_{2}^{*}\right)$ where $t_{2}^{*}$ is the time in which $c_{2}\left(t_{2}^{*}\right)=d_{2}$.

Studying the Jacobian matrix we can see that all the equilibrium points are unstable, in fact:

1) in $P_{1}=(0,0)$ eigenvalues depend to $c_{1}(t), c_{2}(t), d_{1}, d_{2}$

2) in $P_{2}=\left(0, N_{2}^{*}\right)$ one eigenvalue is 0 and the other is $\frac{1}{d_{1}-c_{1}(t)}$

3) in $P_{3}=\left(N_{1}^{*}, 0\right)$ one eigenvalue is 0 and the other is $\frac{1}{d_{2}-c_{2}(t)}$

4) in $P_{4}=\left(N_{1}^{*}, N_{2}^{*}\right)$ both eigenvalues are zero
3) U1 case: In case of U1 (gompertzian growth law) we have $c_{1}(t)=c_{1} e^{\beta_{1} t}, c_{2}(t)=c_{2} e^{\beta_{2} t}$ with $c_{1}, c_{2}, \beta_{1}$ and $\beta_{2}$ constants.

The analytical solutions are:

$$
\begin{aligned}
N_{1} & =e^{\int^{t} c_{1}(s)-d_{1} d s}= \\
& =N_{0,1} e^{\frac{c_{1}}{\beta_{1}} e^{\beta_{1} t}-d_{1} t-\frac{c_{1}}{\beta_{1}}} \\
& =N_{\infty, 1} e^{-r_{1} e^{\frac{t}{N_{\infty}, 1}}-d_{1} t+z_{1}} \\
N_{2} & =e^{\int^{t} c_{2}(s)-d_{2} d s}= \\
& =N_{0,2} e^{\frac{c_{2}}{\beta_{2}} e^{\beta_{2} t}-d_{2} t-\frac{c_{2}}{\beta_{2}}} \\
& =N_{\infty, 2} e^{-r_{2} e^{\frac{t}{N_{\infty}, 2}}-d_{2} t+z_{2}}
\end{aligned}
$$

As concerns equilibrium points we have that $c_{1}(t)=d_{1} \Leftrightarrow c_{1} e^{\beta_{1} t^{*}}=d_{1} \Leftrightarrow t^{*}=\frac{\log \left(\frac{d_{1}}{c_{1}}\right)}{\beta_{1}}$ and $c_{2}(t)=d_{2} \Leftrightarrow c_{2} e^{\beta_{2} t^{*}}=d_{2} \Leftrightarrow t^{*}=\frac{\log \left(\frac{d_{2}}{c_{2}}\right)}{\beta_{2}} ;$ equilibrium points are all unstable.

Studying the limit, we can see that $N_{1}$ tends to zero when $d_{1}>0$ and $N_{2}$ tends to zero when $d_{2}>0$. Re-writing the system using eq. (4) we have:

$$
\left\{\begin{array}{l}
\frac{d N_{1}(t)}{d t}=-r_{1} N_{1}(t) \log \left(\frac{N_{1}}{N_{\infty, 1}}\right)-d_{1} N_{1}(t) \\
\frac{d N_{2}(t)}{d t}=-r_{2} N_{2}(t) \log \left(\frac{N_{2}}{N_{\infty, 2}}\right)-d_{2} N_{2}(t)
\end{array}\right.
$$

In this case both populations admit the solution $N_{.}=N_{\infty, .} e^{-\frac{d \cdot}{r_{\cdot}}} e^{\log \left(\frac{N_{0, .}}{N_{\infty}, .}\right) e^{\frac{d_{\cdot}}{r_{.}}-\frac{r_{. t}}{1-d_{.}}}}$and tend to $N_{\infty, .}^{*}=N_{\infty, .} e^{-\frac{d .}{r_{.}}}$.

\section{GenERAL DESCRIPTION OF THE TWO POPULATIONS MODEL WITH FIXED CARRYING CAPACITY}

In a real situation, of course, both mutations and treatments, sometimes responsible for mutation themselves, are present. Moreover, we expect that the total carrying capacity $N_{\infty}$ will be fixed by physical and/or energetic constraints such as total nutrient present in the body or space available between two membranes. So, if a population is reduced or eliminated, we expect that the other population will increment its carrying capacity. The final system will be:

$\left\{\begin{array}{l}\frac{d N_{1}(t)}{d t}=-r_{1} N_{1} \log \left(\frac{N_{1}}{N_{\infty, 1}}\right)-m N_{1}-d_{1} N_{1} \\ \frac{d N_{2}(t)}{d t}=-r_{2} N_{2} \log \left(\frac{N_{2}}{N_{\infty, 2}}\right)+m N_{1}-d_{2} N_{2}+c N_{2} \\ N_{\infty, 1}^{*}+N_{\infty, 2}^{*}=N_{\infty}\end{array}\right.$

Where $m$ is the mutation term, $d_{1,2}$ the kill rates and $c$ is the term that must be introduced to satisfy the condition $N_{\infty, 1}^{*}+N_{\infty, 2}^{*}=N_{\infty}$. In the 


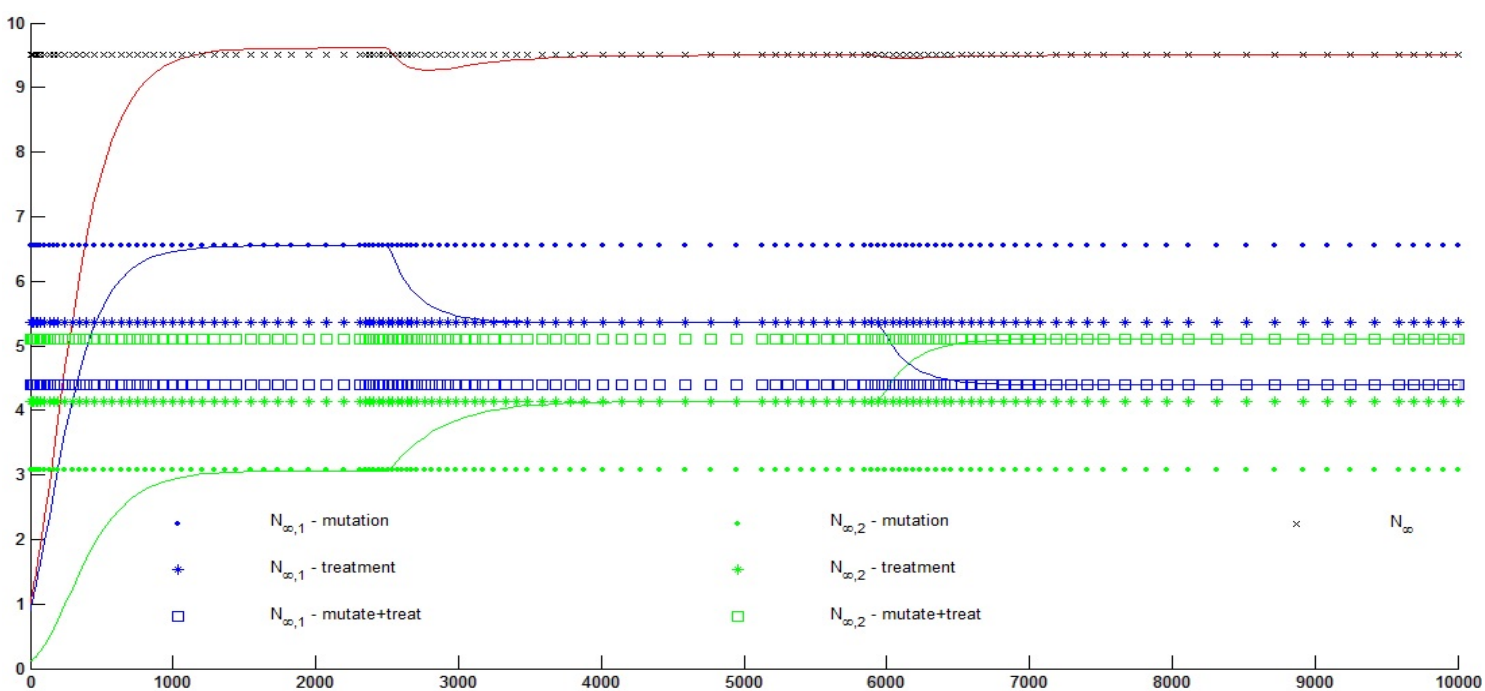

Fig. 1. In the first period $N_{1}$ mutate in $N_{2}$ without treatment; in the second both populations are killed by a treatment but there isn't mutation; in the third period both mutation and treatment are involved. The $c$ term has been set to allow the condition of fixed total carrying capacity.

\begin{tabular}{|c|c|c|c|}
\hline Parameters & Mutation case only & Treatment case only & Mutation with treatment case \\
\hline $\mathrm{N}_{\infty, 1}^{*}$ & $\mathrm{~N}_{\infty, 1} e^{\frac{-m}{r_{1}}}$ & $\mathrm{~N}_{\infty, 1} e^{\frac{-d_{1}}{r_{1}}}$ & $\mathrm{~N}_{\infty, 1} e^{\frac{-\left(m+d_{1}\right)}{r_{1}}}$ \\
\hline & $c+m \frac{N_{1}^{*}}{N_{2}^{*}}$ & $-\underline{d_{2}-c}$ & $d_{2}-c-m \frac{N_{1}^{*}}{N_{2}^{*}}$ \\
\hline $\mathrm{N}_{\infty, 2}^{*}$ & $\mathrm{~N}_{\infty, 2} e^{r_{2}}$ & $\mathrm{~N}_{\infty, 2} e^{-\frac{2}{r_{2}}}$ & $\mathrm{~N}_{\infty, 2} e^{-}$ \\
\hline c & $\mathrm{r}_{2} \log \left(\frac{N_{\infty}-N_{\infty, 1} e^{-\frac{m}{r_{1}}}}{N_{\infty, 2}}\right)-m \frac{N_{1}^{*}}{N_{2}^{*}}$ & $\mathrm{r}_{2} \log \left(\frac{N_{\infty}-N_{\infty, 1} e^{-\frac{d_{1}}{r_{1}}}}{N_{\infty, 2}}\right)-d_{1}$ & $\mathrm{r}_{2} \log \left(\frac{N_{\infty}-N_{\infty, 1} e^{-\frac{m+d_{1}}{r_{1}}}}{N_{\infty, 2}}\right)-m \frac{N_{1}^{*}}{N_{2}^{*}}-d_{1}$ \\
\hline
\end{tabular}

table we summarize how the carrying capacities change during mutation, treatment and treatment with mutation respectively; we can also calculate how $c$ will vary to allow $N_{\infty}$ to be constant, i.e. the growth spurt of $N_{2}$ in the event of death or drastic reduction of $N_{1}$. This approach could be useful in case of treatments that affects only population 1: we can simulate both the rapid increase of $N_{2}$ and the rapid decrease of $N_{1}$ (e.g. surgical removal of primary tumor and rapid increase in metastasis). Further simulations will seek to maximize the $d_{2}$ value in order to eradicate the second population too.

\section{Conclusions}

The PUN approach provides both interesting analytical results and an easy way to model the tumor growth also in more realistic, non homogeneous cancers. A two populations model could explain the behaviour of the cancer during treatment and allows us to simulate different scenarios in order to choose the best therapy for the patient. Further investigation is required to estimate the parameters values using real data and to understand more into details the role of the critical times $t^{*}$, in order to find, whenever possible, the 'best time for the therapy'. Moreover, in real situations $d$. and $m$ are time dependent and the functions $d .(t)$ and $m(t)$ will be considered instead of constant parameters.

\section{REFERENCES}

[1] Ahmed M, Li LC., Adaptation and clonal selection models of castration-resistant prostate cancer: Current perspective. IJU Vol.20 (2013) 362-371.

[2] Finones RR, Yeargin J, Lee M et al. Early Human Prostate Adenocarcinomas Harbor Androgen-Independent Cancer Cells. PLOS ONE Vol. 8 (2013) e 74438

[3] L. Hanin, S. Bunimovich-Mendrazitsky, Reconstruction of the natural history of metastatic cancer and assessment of the effects of surgery: Gompertzian growth of the primary tumor, Mathematical Biosciences, Vol.247 (2014) 47-58.

[4] C.Guiot, P.G.Degiorgis,P.P.Delsanto,P.Gabriele,T.S.Deisboeck, Does Tumor growth follow a 'universal law'?, Journal of Theoretical Biology, Vol.225 (2003) 147-151.

[5] P.Castorina, P.P.Delsanto, C.Guiot, A Classification Scheme for Phenomenological Universalities in Growth Problems in Physics and Other Sciences, Physical Review Letters Vol. 96 Issue: 18 